\title{
REVISIÓN
}

REVIEW

\section{PHASA: TODO SOBRE LA ARCILLA COMESTIBLE}

\section{PHASA: EVERYTHING ABOUT EDIBLE CLAY}

\author{
Giovanny Fernando Ramirez Alanoca ${ }^{1}$, Victor Joaquin Copa Gisbert ${ }^{2}$.
}

\section{RESUMEN}

1 Estudiante de 4to año de medicina, Universidad Mayor de San Andrés, La Paz, Bolivia.

2 Universidad Mayor de San Andrés Facultad de Medicina Enfermería Nutrición Tecnología Médica: La Paz, BO.

Correspondencia a: Giovanny Fernando Ramirez Alanoca

Correo electrónico: ramirezgiovanny69@gmail. com

Teléfono: 22833648 +59168064192

ORCID: https://orcid.org/00000001-9901-2519

https://orcid.org/0000-00032326-1150

Palabras clave: arcilla, geofagia, medicina tradicional, montmorillonita.

Keywords: clay, pica, medicine traditional, bentonite.
Procedencia y arbitraje: no comisionado, sometido a arbitraje externo.

Recibido para publicación: 1 de abril de 2020

Aceptado para publicación: 9 de enero de 2021

Citar como:

Ramirez Alanoca GF, Copa

Gisbert VJ. Todo sobre la arcilla

comestible. Rev Cient Cienc

Med 2020; 23 (2): 240-246

La phasa (conocida así entre los pobladores aymaras) es un tipo de arcilla comestible que se encuentra en los suelos, empleada como medicina tradicional en la mayoría de los continentes, principalmente para aliviar o tratar patologías gastrointestinales e infecciones cutáneas. Su consumo se remonta muchos siglos atrás; existe evidencia del uso de este elemento en las Placas de arcilla de Nippur en Mesopotamia (2500 años a. C.).

Develar las propiedades bio-físico-químicas de las arcillas comestibles está permitiendo a la ciencia explicar las propiedades terapéuticas que posee y así confirmar los grandes avances en la medicina alternativa que tuvieron nuestros antepasados.

El uso de la phasa no se limita a la medicina, sino también a muchas otras ramas de la ciencia. En los últimos 10 años se ha experimentado en la agricultura, veterinaria, incluso en el cuidado del medio ambiente dando resultados muy gratificantes y prometedores.

\section{ABSTRACT}

Phasa, an Aymara's language word, is a type of edible clay found in soils; which is used in traditional medicine worldwide mainly to relieve or treat gastrointestinal pathologies and skin infections. Its consumption dates back many centuries, even millennia ago; there is evidence of the use of this element in the clay plates of Nippur in Mesopotamia (2500 years BC).

Revealing the bio-physical-chemical properties of edible clay has allowed science to explain their healing and therapeutic properties and confirms the great advances our ancestors got.

The use of phasa is not limited to medicine, it is also used in many other sciences; in the last 10 years it has been used in agriculture and veterinary medicine, getting gratifying results.

\section{INTRODUCCIÓN}

El consumo de la phasa (vocablo aymara), chaco (vocablo quechua) o simplemente arcilla comestible; junto a sus minerales, es una práctica en la medicina que inició muchos siglos atrás, en diversas culturas del mundo. El uso de la arcilla comestible por parte de los humanos fue influenciado por aspectos biológicos (nutricional, fisiológico), psicológicos y sociales (religión, cultura, rituales, hambruna, pobreza, etc.); los cuales motivaron a que las personas encontraran las grandes propiedades que posee dicho derivado mineral.

Bolivia al ser un país rico en costumbres heredadas de sus antepasados, no está exenta del uso de medicina alternativa como tratamiento en diversas patologías en el cotidiano vivir de su población. En Bolivia hay dos principales departamentos que proveen la phasa: Oruro y La Paz, por ello se realiza una revisión bibliográfica minuciosa para validar nuevas alternativas de tratamiento de origen natural y traducirlo a posteriori al conocimiento con bases científicas.

\section{METODOLOGÍA}

Se presenta una revisión de tipo descriptiva. Se realizó la búsqueda bibliográfica en: GOOGLE ACADÉMICO, LILACS, MEDLINE PLUS, MEDIAGRAPHIC, PUBMED, SCIELO; con los siguientes criterios de selección: arcilla comestible AND salud, chaco, edible clay AND health, geofagia AND salud, medical use of clay, phasa AND salud. Se encontraron 62 resultados, fueron escogidos 42 por su relevancia dentro de la medicina y fueron excluidos aquellos que se enfocaron en el uso no médico de la arcilla y sobrepasaban la antigüedad mayor a los 15 años, exceptuando 2 por tener índole histórica. 


\section{DESARROLLO}

"En su cosmovisión (de los Kallawayas), la definición de salud proviene de una analogía entre el cuerpo humano y la tierra, los animales, las comunidades, la realización personal y el respeto de las costumbres. Una visión que toma auge en el mundo occidental" ${ }^{1}$.

\section{Historia}

El uso de la arcilla por la humanidad (desde que se pudo documentar), se remonta a la época de las Placas de arcilla de Nippur (2500 años a. C.) y del Papiro de Ebers (1600 años a.C.) $)^{2}$, en las cuales se destaca su empleo en la alimentación y como agente anti-infeccioso en lesiones cutáneas; asimismo se menciona la atención que le brindaron hombres de ciencia como Dioscórides, quien describe el uso de tierras medicinales en su obra "De materia médica". L. Giessler la empleó en la peste; por su lado, Kunza y Vogel descubrieron su capacidad de adsorción y retención de toxinas intestinales ${ }^{3}$.

La phasa es un tipo de arcilla constituida por agregados de silicato; su consumo se conoce como geofagia y existe evidencia del consumo de la phasa en animales y humanos tanto en África, Centroamérica, Europa y Sudamérica ${ }^{4}$. Durante los siglos XVI y XVII el consumo de arcilla se extendió entre la nobleza europea que tuvo como fuente de arcilla a los búcaros (vasijas de arcilla), los cuales se ven representados en la literatura y pintura española de la época. En Ecuador se mezclaba arcilla con agua para ser bebida por una comunidad indígena; los Tigua $^{5}$. En Perú la geofagia entre sus pobladores inició hace 500 años debido a la agricultura, especialmente con el consumo de la papa (Solanum tuberosum). En Bolivia se encontraron yacimientos de arcilla en Achocalla, Mocomoco y Andamarca, la cual es extraída de socavones, secada y posteriormente comercializada para su consumo ${ }^{6}$. Otros autores aseguran que la geofagia inició en la región Central de los Andes junto al pastoreo de camélidos; quienes lamían arcilla rica en filosilicatos hidratados y aliviaban el malestar generado por las fitotoxinas presentes en muchas plantas. La evidencia más antigua conocida de geofagia proviene de un estudio de coprolitos humanos del periodo arcaico en la cuenca del lago Titicaca, donde se encontraron muestras con una antigüedad de hasta tres milenios; asimismo se encontró evidencia de geofagia en Tiwanaku (Bolivia) y en el norte de Chile, el upi (vocablo con el cual se conoce a la phasa en esa región) ${ }^{7}$. Además, existe evidencia científica de que las arcillas estuvieron involucradas en la formación de biomoléculas de la Tierra primitiva al servir como catalizadores en la formación de oligómeros de ARN (ácido ribonucleico) demostrando su importancia biológica ${ }^{8}$.

\section{Estructura química y propiedades físicas}

La phasa es un tipo de arcilla con una estructura básica del tipo 2:1, formada por dos capas de tetraedros de Si4t, generando internamente una capa octaédrica de Al3+. En la caracterización de dos tipos de phasa provenientes de dos departamentos de Bolivia (Oruro y La Paz), se observó a nivel fisicoquímico que la proveniente de La Paz presentaba un mayor carácter sódico-potásico, es decir mejor neutralización de ácidos e hidratación; propiedades que la hacen una sustancia adecuada para su uso en la medicina. La capacidad de intercambio catiónico de la arcilla incrementa la superficie de absorción y el potencial detoxificador con las toxinas orgánicas ${ }^{9}$.

En la caracterización química de las arcillas con fluorescencia de Rayos $\mathrm{X}$ se detectó la presencia de al menos 18 compuestos químicos entre ellos: Silicio, Aluminio, Potasio, Hierro, Calcio, Titanio, Estroncio, Zirconio, Magnesio, Zinc, Fósforo, Sodio, Rubidio, Cobre, Bario, Galio y Niobio (ordenados de mayor a menor según su nivel de concentración) ${ }^{10}$.

Posibles aplicaciones de la Phasa en la medicina (ver tabla 1)

Se documentó el uso de la arcilla de manera tópica, en especial para combatir infecciones de la piel por sus propiedades adsortivas ${ }^{11}$. Se reportaron propiedades antibacterianas en el tratamiento de Úlcera Buruli (causada por Mycobacterium ulcerans) en niños de África según los resultados de un estudio experimental in vitro; asimismo una variedad de arcilla tuvo efecto bacteriostático contra bacterias Gram negativas ${ }^{12}$. Otra revisión señala que a dosis altas resulta ser bactericida con Escherichia coli, pero ninguno en Staphyfococcus aureus meticilino resistente (MRSA), lo cual lleva a inferir que la arcilla tiene mejores efectos bactericidas/ bacteriostáticos en Gram negativas que en Gram positivas. Se cree que su acción antibacteriana tienecomo fundamento principal el intercambio de iones de potasio entre la arcilla y las bacterias, 
por lo que destruiría la estructura bacteriana ${ }^{13}$. Las principales funciones de los metales de la arcilla son la creación de radicales de oxígeno, los cuales dañan a las bacterias. Los metales más importantes por su acción antibacteriana son el aluminio y el hierro, ya que el aluminio deforma la envoltura celular bacteriana y el hierro facilita la oxidación de sus componentes, además, una serie de reacciones químicas dañan el DNA de la bacteria, perpetuando su muerte ${ }^{14}$. Asimismo, estudios demuestran que el hierro que se encuentra en la arcilla es responsable de la formación de especies reactivas de oxígeno (EROS) que predispone al estrés oxidativo de la bacteria ${ }^{15}$. En estudios recientes, se evidenció que el consumo de arcilla comestible en mujeres embarazadas o durante la lactancia materna es beneficioso, ya que es fuente de gran cantidad de electrolitos. También se utilizó en el norte de América junto al consumo de maíz en poblaciones indígenas originarias de la región ${ }^{16}$. Por último, según un estudio experimental in vitro, el uso de arcilla comestible mejoró la absorción de algunos medicamentos como la trimetoprima ${ }^{17}$.

Riesgos y efectos adversos del consumo de

\section{Phasa y otras arcillas}

Debido a su origen, el consumo de la arcilla comestible es altamente predisponente a presentar riesgos a la salud, principalmente por la presencia de arcillas contaminadas con microorganismos patógenos y metales pesados $\left(\mathrm{Pd}, \mathrm{Hg}, \mathrm{Cd}\right.$, etc.) ${ }^{18}$. A nivel gastrointestinal se ha documentado que produce: estreñimiento, parasitosis y malabsorción de medicamentos tomados simultáneamente (diosmectita, montmorillonita) ${ }^{19}$.

El uso de las arcillas de manera indiscriminada puede traer consigo la introducción de DNA exógeno tanto en células eucariotas como en procariotas y el rompimiento de cadenas de DNA. Los dos anteriores mecanismos serían factores de riesgo tanto para la producción de resistencia a antimicrobianos en bacterias (principalmente Escherichia coli) como para la mutación causante del cáncer en organismos superiores. Los metales pesados no influirían en la mutación genética. Además se evidenció que hay mejor transporte de DNA exógeno al interior de la bacteria cuando esta está sometida a la fricción (como en el tubo gastrointestinal) ${ }^{20}$. Asimismo se demostró que el consumo de

Tabla 1. Propiedades, de la Phasa y sus efectos en el organismo humano

\begin{tabular}{|c|c|c|}
\hline Propiedad de la Phasa & Efecto en el Organismo & Uso terapéutico \\
\hline $\begin{array}{l}\text { Capacidad absorbente } \\
\text { Detoxificante }\end{array}$ & $\begin{array}{l}\text { Interacción con sustancias polares } \\
\text { (agua y toxinas) y absorción de áci- } \\
\text { dos biliares o enzimas. Capacidad } \\
\text { de intercambio catiónico. }\end{array}$ & $\begin{array}{l}\text { Enterocolitis, ptialismo en el emba- } \\
\text { razo y diarrea. }\end{array}$ \\
\hline Hidrófilo & Mejor dilución en agua. & $\begin{array}{l}\text { Facilita la administración por vía } \\
\text { oral. }\end{array}$ \\
\hline Activador de la coagulación & $\begin{array}{l}\text { Unión a cationes }(\mathrm{H}+) \text {, Activación } \\
\text { de los factores de la coagulación. }\end{array}$ & Hemorragias cutáneas persistentes. \\
\hline Alcalinizante & $\begin{array}{l}\text { Intercambio de } \mathrm{H+} \text {, alcaliniza el pH } \\
\text { ácido del estómago. }\end{array}$ & Gastritis, úlceras gastroduodenales. \\
\hline Bactericida & $\begin{array}{l}\text { Elementos reducibles intercambia- } \\
\text { bles }(\mathrm{Fe} 2+) \text {. } \\
\text { Intercambio de iones de potasio } \\
\text { entre la arcilla y las bacterias. }\end{array}$ & Enterocolitis, infecciones cutáneas. \\
\hline Efecto anti-flogósico & $\begin{array}{l}\text { Alta capacidad de retención de } \\
\text { calor. }\end{array}$ & $\begin{array}{l}\text { Infecciones cutáneas, quemaduras } \\
\text { de primer grado. }\end{array}$ \\
\hline Suplemento nutricional & $\begin{array}{l}\text { Acción liberadora de micronutrien- } \\
\text { tes u oligoelementos. }\end{array}$ & Anemia ferropénica. \\
\hline
\end{tabular}

Fuente. Elaboración propia 
arcillas comestibles durante 28 días produjo cambios en el perfil lipídico de ratas WISTAR, elevación del CRR (cardiac risk ratio), CRIII (Castelli's risk index II) y AC (atherogenic coefficient) ${ }^{21}$; herramientas empleadas para evaluar el riesgo coronario.

Comercialización de la Phasa y otros tipos de arcilla

En el sistema de clasificación anatómico, terapéutico, químico (ATC) figura bajo el nombre de BEDELIX (código ATC: A07BC05), que tiene como principio activo a la arcilla montmorillonita beidellitica, indicada para colopatía funcional (o síndrome de intestino irritable) ${ }^{22}$.

En Boliviaparticularmenteen el departamento de La Paz, la phasa es comercializada sin prescripción médica, es decir, de venta libre en tiendas especializadas en medicinas tradicionales y demás elementos relacionados con la viva cultura de la región denominadas "chiflerías".

\section{DISCUSIÓN}

La geofagia es una práctica empleada tanto por animales como por humanos desde tiempos inmemoriales por la capacidad de la arcilla de inhibir toxinas vegetales, como suplemento alimenticio y también con fines estéticos ${ }^{23,24}$.

Es importante mencionar que poco se ha investigado de las propiedades de la phasa en Bolivia y países aledaños, debido a ello se obvian algunos efectos adversos o contraindicaciones de su uso. Las grandes cantidades de plomo y selenio en la arcilla de consumo pueden resultar tóxicas para el consumidor ${ }^{25}$. Otro riesgo atribuible a la geofagia de arcilla es la calidad higiénica de su recolección, procesamiento y posterior distribución; la cual tiene como consecuencia la adquisición de enfermedades infecciosas, principalmente de tipo parasitarias. Estas parasitosis tienen por agente etiológico a geohelmintos y otros parásitos que colonizan el tubo gastrointestinal, siendo de mayor frecuencia en niños, desencadenando desde diarreas hasta neumonías de tipo eosinofílicas 26-28.

Se realizaron trabajos experimentales que empleaban la arcilla o tierras similares en la terapéutica; las prácticas derivadas de esta reciben el nombre de geoterapia, teniendo buenos resultados en el tratamiento de infecciones cutáneas (la arcilla posee actividad bactericida), quemaduras (aparte de su acción bactericida, acelera el tiempo de cicatrización en el $95 \%$ de los sujetos) y en la farmacología (se emplea la arcilla y otras tierras como excipientes farmacológicos) ${ }^{29-32}$.

Jiao et al., demostró que la montmorillonita cargada con cobre y zinc aumentó los niveles de absorción de los mismos y redujo la concentración de citocinas inflamatorias (IL1 beta, IL-6 y FNT-alfa) en el intestino delgado (yeyuno e íleon) ${ }^{33}$. Pese a sus reacciones adversas el consumo de montmorillonita de calcio tiene una seguridad relativa en sujetos humanos ${ }^{34}$. Particularmente en niños, el uso de montmorillonita de calcio redujo las concentraciones de aflatoxinas que se encuentran en los suplementos nutricionales hechos en base a maní o maíz ${ }^{35}$, tal como otra investigación demostró que la suplementación de bentonita de calcio con cumarina mejora los daños causados por la aflatoxina B1 al reducir su biodisponibilidad, efecto que se presenta no solo en la especie humana sino en variedad de mamíferos ${ }^{36,37}$. En el ámbito dermatológico, como tratamiento del acné, piel grasa o seca, ya que son de fácil aplicabilidad, remoción y presentan mucha inocuidad. Por otra parte, una preparación de organoarcilla fue estadísticamente mejor que otros preparados como kaolina o bentonita en la prevención tópica de la dermatitis provocada por hiedra venenosa sintética ${ }^{38,39}$. La dermatitis del pañal infantil tuvo mejor curación al aplicar cremas en base a bentonita en comparación a la caléndula ${ }^{40}$. Una especial mención son las investigaciones llevadas a cabo en relación al tratamiento sintomático del Síndrome de Intestino Irritable, los cuales evidenciaron que la montmorillonita beidellitica reduce el dolor abdominal y las molestias, lo que fue mayor en el grupo con constipación que en el grupo con diarrea ${ }^{41,42}$.

\section{CONCLUSIONES}

El uso de la phasa en la medicina ha sobrepasado lo meramente empírico, gracias a los avances de la tecnología y los métodos de experimentación, equipos de medición y análisis de los distintos tipos de arcillas 
comestibles han permitido sustentar la base bio-físico-química de los distintos tipos de usos que se las atribuye. Se encontró un amplio conjunto de propiedades que posee la phasa, lo cual justifica su consumo por las antiguas culturas como medicina alternativa. Entre las propiedades que destacan están las siguientes: su interacción con los cationes de $\mathrm{H}+$ (principal razón de consumo con el propósito de apaciguar los síntomas de la úlcera péptica, una enfermedad muy prevalente en nuestro medio); por otro lado tenemos la neutralización de toxinas, la principal causa de consumo de phasa por parte de los animales (en especial en los camélidos). Entre los artículos citados en la bibliografía se pudo demostrar el uso de la arcilla por vía tópica como antibacteriana en otros continentes. La evidente disponibilidad de la arcilla le ha valido para ser objeto de varias investigaciones, para su uso en otros ámbitos de la ciencia; por ejemplo en la avicultura está relacionado con la reducción de cepas con resistencia antimicrobiana a fármacos, lo que favorece a la crianza de aves de corral para el posterior consumo humano. Si bien existen evidencias sobre los efectos a favor en la salud, no existen suficientes investigaciones sobre los efectos adversos que podrían ser contraproducentes para la salud de las personas. Se espera que los estudios continúen con el afán de enriquecer conocimientos y poner en evidencia la indiscutible sapiencia de los antepasados con relación a la phasa y sus propiedades para poder usarla en la terapéutica de enfermedades prevalentes en distintas regiones del mundo. El reto es continuar investigando no solo las propiedades terapéuticas y efectos adversos de estas arcillas, sino de muchas otras que forman parte de la amplia gama de la medicina tradicional andina en Bolivia y en el continente sudamericano; realizar investigaciones en la población, debido a que en la revisión de la literatura se evidenció la carencia de estudios in vivo, los cuales no necesariamente deben ser de carácter invasivo, sino más bien ser exámenes laboratoriales y de gabinete. 


\section{REFERENCIAS}

1. Alarcón C, Philco JN. Turismo de salud: revalorización de la medicina tradicional Kallawaya en el municipio de Charazani [Thesis]. La Paz (BOL): Universidad mayor de San Andrés; 2018. [Acceso 18 de agosto de 2019]. Disponible en: https://docplayer.es/114392982-Vniversidad-mayor-de-san-andres-facultad-de-humanidades-y-ciencias-de-la-educacion-carrera-de-turismo.html

2. Gomes CSF. Healing and edible clays: a review of basic concepts, benefits and risks. Environ Geochem Health. [Internet] 2018; [Acceso 26 de junio de 2020] 40(5): 1739-1765; Disponible en: https:/pubmed.nc6i.nlm.nif.gov/28150053/

3. Herguedas A. Estudio clínico analítico sobre el uso de la arcilla para uso interno. Natura Medicatrix. [Internet] [Acceso 25 de junio de 2020] (55): 16-25 Disponible en: https:// dialnet. unirioja.es/servlet/articulo? codigo $=4989280$

4. Mamani Q, Carlos J. Determinación del efecto de tres niveles de phasa (Bicarbonato doble de calcio) en la calidad del huevo en aves de línea isa brown fase postura pico en la provincia Loayza - La Paz [Thesis]. La Paz (BOL): Universidad Mayor de San Andrés; 2014. Acceso 18 de agosto de 2019. Disponible en: https://repositorio.umsa.6o/Gitstream/handle $/ 123456789 / 4261 /$ T-1816.pdf? sequence $=1$ Q Z is Allowed $=y$

5. García M, Álvarez B. Origen y distribución de arcillas utilizadas en la fabricación de búcaros: bucarofagia en la Edad Moderna. Physis Terrae. [Internet] 2019; [Acceso 24 de junio de 2020] 1(1):57-71 Disponible en: https://revistas.uminho.pt/index.php/physisterrae/article/view/326

6. Castillo O, Frisancho O. El "chaco": arcilla medicinal comestible del altiplano peruano y sus propiedades en la patologla digestiva. Rev. gastroenterol. [Internet] 2015 [Acceso 18 de agosto de 2019] 35(1): 97-9 Disponible en:http://www.scielo.org.pe/scielo.php?script=sci_arttext\&ipid $=$ S1022-51292015000100013 Z Ing $=$ es .

7. Browman DL. Tierras comestibles de la Cuenca del Titicaca: Geofagia en la prehistoria boliviana. Estudios atacameños [Internet] 2004; [Acceso 18 de agosto de 2019] (28):133-41. Disponible en: https://scielo.conicyt.cl/scielo. php?script=sci_arttext\&pid=S0718-10432004002800011

8. Ertem G, Zhehong G. Role of preparation method on the extent of montmorillonite catalysis for oligomer formation. Applied Clay Science. [Internet] 2014; [Acceso 17 de agosto de 2019] 101:90-3 Disponible en: https://www.sciencedirect.com/science/article/abs/pii/S0169131714002774

9. Johns T. Technological methods of detoxification. [publisher unknow]. The Origins of Human Diet and Medicine: Chemical Ecology. 1ra ed. Arizona: University of Arizona Press; [Internet] c1996. p. 71-100. [Acceso 19 de agosto de 2019] Disponible en: https://6ooks.google.com.60/6ooks. id =D -

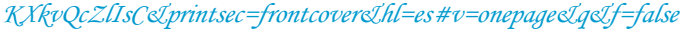

10. Ticona $W$, Blanco $M$, Cabrera S. CARACTERIZACION QUIMICA MINERALOGICA ESTRUCTURAL DE DOS ARCILLAS BOLIMANAS. Rev. Bol. Quim. [Internet] 2006; [Acceso 27 de junio de 2020] 23(1): 71-6; Disponible en: $\quad$ http://www.scielo.org.6o/scielo.php?script=sci_arttex-

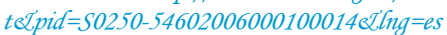

11. Kikouama OJ, Balde L. From edible clay to a clay-containing formulation for optimization of oral delivery of some trace elements: a review. Int J Food Sci Nutr. [Internet] 2010; [Acceso 17 de enero de 2020] 61(8): 803-822; Disponible en: https://pubmed.nc6inlm.nih.gov/20528584/

12. Zarate-Reyes L, Lopez-Pacheco C, Nieto-Camacho A, et al. Antibacterial clay against gram-negative antibiotic resistant bacteria. J Hazard Mater. [Internet] 2018; [Acceso 17 de enero de 2020] 342:625-632 Disponible en: sciencedirect.

\section{com/science/article/abs/pii/S0304389417306787}

13. Williams L, Haydel S. Evaluation of the medicinal use of clay minerals as antibacterial agents. Int Geol Rev. [Internet] 2010; [Acceso 15 de enero de 2020] 52(7/8):74570 Disponible en: https:/www.nc6inlm.nif.gov/pmc/articles/ PMC2904249/\#: :text=The\%20highly\%20adsorptive\%20properties\%20of, alt hough\%20they\%20are\%20not\%20antibacterial. $\mathcal{2}$ tex$t=$ The \%20two\%20French\%20green\%20clays, on \%20the\%206acterial\%20populations\%20tested.

14. Haydel SE, Remenih CM, Williams LB. Broad-spectrum in vitro antibacterial activities of clay minerals against antibiotic-susceptible and antibiotic-resistant bacterial pathogens. J Antimicrob Chemother. [Internet] 2008; [Acceso 15 de enero de 2020] 61(2): 353-361; Disponible en: https:// pubmed.nc6inlm.nih.gov/18070832/

15. Morrison, K, Misra R, Williams L. Unearthing the Antibacterial Mechanism of Medicinal Clay: A Geochemical Approach to Combating Antibiotic Resistance. Sci Rep. [Internet] 2016; [Acceso 25 de agosto de 2019] 6(19043):1-13 Disponible en: https://www.nature.com/articles/srep19043

16. Ogah SPI, Akpomie TM, Nwite EO. Estimation of Some Essential Metallic Elements in Edible Clay from Enyigba in Ebonyi State of Nigeria Using AAS and Experiments with Rabbits. ACSJ. [Internet] 2016; [Acceso 26 de enero de 2020] 16(4):1-15; Disponible en: fitp://wrwwsciencedomainorg/abstract/16260

17. Okhuelegbe E, Amara I. IN VITRO INTERACTION BETWEEN TRIMETHOPRIM AND SOME ANTACIDS AND EDIBLE CLAY. Pak J Pharm. [Internet] 2012; [Acceso 18 de agosto de 2019] 25(1/2):3-7; Disponible en: https:/ /www.semanticscholar. org/paper/IN-VITRO-IDTERACTION-BETWEEN-TRIMETHOPRIM-AND-SOME-OKhueleg6e-Amara/Oae5aad131c41afc1e86adc5786dacad4f71e632

18. LONDOÑO-FRANCO LUIS FERNANDO, LONDOÑO-MUÑOZ PAULA TATIANA, MUÑOZ-GARCÍA FABIÁN GERARDO. LOS RIESGOS DE LOS METALES PESADOS EN LA SALUD HUMANA Y ANIMAL. Rev.Bio.Agro. [Internet] 2016; [Acceso 26 de enero de 2020] 14(2):145-153; Disponible en: http://www.scielo.org.co/scielo.php?script=sci_arttextatpid $=$ S1692-35612016000200017 R'ing=en.

19. Finkelman R. Health Benefits of Geologic Materials and Geologic Processes. En: William R, editor. New Methods and Studies. Toronto: CRC Press; [internet] 2012; [ACceso 22 de enero de 2020] p16-23; Disponible en: https:// 6ooks.google.com.ec/6ooks? id =9918DgA AQB AJ L Printsec = frontcoverci $h l=e s \# v=$ onepage 2 iq $2 \mathcal{L} f=$ false

20. González-Tortuero E, Rodríguez-Beltrán J, Radek $R$, Blázquez J, Rodríguez-Rojas A. Clay-induced DNA breaks as a path for genetic diversity, antibiotic resistance, and asbestos carcinogenesis. Sci Rep. [Internet] 2018; [Acceso 22 de enero 2020] 8(1):8504; Disponible en: https://pubmed.nc6i.n/m. nif.gov/29855603/

21. Ikewuchi CC, Ikewuchi JC, Ezeka UK, Ifeanacho MO. Effect of "edible clay" (takere) suspension on serum lipid profiles and atherogenic indices of normal Wistar rats. Food Sci Nutr. [Internet] 2019; [Acceso 18 de agosto de 2019] 7(3):977-986; Disponible en: https:/pubmed.nc6inlm. nif.gov/30918640/

22. whocc.no. Noruega: WHO Collaboratting Centre for drug Statistics Methodology; [Internet] 2019 [Acceso 18 de agosto de 2019] Disponible en: https://www.whocc.no/ atc_ddd indexl? code $=$ A07BCO5

23. Buroni J, Buroni M. Las patologías que plasm6 Velázquez en 'Las Meninas". Alm Med Cult. [Internet] 2016; [Acceso 18 de julio de 2020] 2(3): 42-53; Disponible en: 
http://www.almarevista.com/revista/arte-y-medicina/Las-patologiasque-plasmo-velazquez-en-las-meninas/

24. Pijoan M. La automedicación animal y su interés farmacologico. OFFARM. [Internet] 2003; [Acceso 18 de julio de 2020] 22(7): 84-92; Disponible en: https://www.elsevier. es/es-revista-offarm-4-articulo-La-automedicacion-animal-su-interes-13050012

25. Chui $H$, Acosta $D$, Olivera de Lescano $P$, Huamán $G$. CHEMICAL AND MINERALOGICAL CHARACTERIZATION OF MACHU RUMI, A CLAY WITH GEOFAGIC USES BY RESIDENTS OF CHIMPAILAHUATA COMMUNITY OF AZANGARO DISTRICT, PUNO, PERU. Rev Bol Quim. [Internet] 2016; [Acceso 18 de julio de 2020] 33(5): 190-195; Disponible en: http://www.scielo.org.6o/scielo.php?script=sci_abstractoL.pid

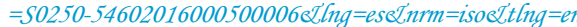

26. Vázquez O, Campos T, López N, Martínez - Barbabosa I, Romero M. Neumonía eosinofilica secundaria a larva migrans visceral en un niño. Rev Mex Patol Clin. [Internet] 2001; [Acceso 18 de julio de 2020] 48(3): 156-160; Disponible en: https://www.medigraphic.com/cgi-6in/new/resumen. cgi?IDARTICU $\mathcal{L O}=5835$

27. Chávez F, Saraí $C$, Benavides D, Carrillo S, Chávez $M$. Parasitosis zoon6ticas presentes en el ambiente. Rev Lat Amb Cien. [Internet] 2018; 9 (21): 1416 - 1424. [Acceso $18 \mathrm{de}$ julio de 2020] p: 9 Disponible en: http://cmas.siu.6uap.mx/portal_pprd/work/sites/rlac/resources/LocalContent/86/2/9(21)-101.pdf

28. Melgar M, Mendaro A, Pizzorno N, Poloni A, Rébora M, Delfino M. Prevalencia de parasitosis intestinal en niños. An Facultad Med. [Internet] 2016; 3 (1): [Acceso 18 de julio de 2020] 3(1): 23-29; Disponible en: http://www.anfamed.edu.uy/ index.php/rev/article/view/180

29. Rodríguez R, González j. Métodos alternativos para el tratamiento de pacientes con heridas infectadas. Medisan. [Internet] 2011; [Acceso 18 de julio de 2020] 15(4) 503-514; Disponible en: https://www.researchigate.net/publication/262740469 Metodos alternativos para el tratamiento de pacientes_con_heridas_infectadas

30. Yebra - Rodriguez A, Cerezo P. Usos farmacéuticos de los minerales de a arcilla. Ens Cien Tier. [Internet] 2018, [Acceso 18 de julio de 2020] 26(3): 289-294; Disponible en: https://www. raco.cat/index.php/ECT/article/view/343165

31. Saz P, Saz S. Utilización terapéutica de la arcilla. Res Gate. [Internet] 2013; [Acceso 18 de julio de 2020] 21: 279-287; Disponible en: https://www.researchgate.net/publication/298861012 Vtilizacion terapeutica_de la arcilla\#: : text=arcilla\%2C\%20arcilla\%206entonita\%20act\%C3\%BAa\%20muy, contra\%20de\%20C\% C3\% A1ndida\%20(116). Q Z text=tanto\%20son\%20 muy\%20eficaces\%20contra, abscesos\%20y\%20seborrea\%20(117)

32. Arévalo Rivas LD, Rodríguez Valles $J$, Ruberto Elías $D$. Efecto de la arcilla medicinal en quemaduras de segundo grado en pacientes del Hospital Nacional Alberto Sabogal Sologuren. Rev Per Med Int. [Internet] 2016; [Acceso 18 de julio de 2020] 1(1): 25-30; Disponible en: http://rpmi.pe/ wp-content/uploads/2016/04/v01n01-25-30.pdf

33. Jiao $L F$, Zhang $Q H, W u H$, et al. Influences of Copper/ Zinc-Loaded Montmorillonite on Growth Performance,
Mineral Retention, Intestinal Morphology, Mucosa Antioxidant Capacity, and Cytokine Contents in Weaned Piglets. Biol Trace Elem Res. [Internet] 2018; [Acceso 18 de julio de 2020] 185(2): 356-363; Disponible en: https://pubmed.nc6inlm. nif.gov/29468611/

34. Wang JS, Luo H, Billam M, et al. Short-term safety evaluation of processed calcium montmorillonite clay (NovaSil) in humans. Food Addit Contam. [Internet] 2005; [Acceso 18 de julio de 2020] 22(3):270-279; Disponible en: https:// pubmed.nc6inlm.nih.gov/16019795/

35. Mitchell NJ, Kumi J, Aleser M, et al. Short-term safety and efficacy of calcium montmorillonite clay (UPSN) in children. Am J Trop Med Hyg. [Internet] 2014; [Acceso 18 de julio de 2020] 91(4):777-785; Disponible en: https:/pubmed. nc6i.nlm.nif.gov/25135766/

36. Hassan AA, Abu Hafsa SH, Elghandour MMMY, Kanth Reddy PR, Monroy JC, Salem AZM. Dietary Supplementation with sodium bentonite and coumarin alleviates the toxicity of aflatoxin B1 in rabbits. Toxicon. [Internet] 2019; [Acceso 19 de julio de 2020] 171: 35-42; Disponible en: https://pubmed.nc6inlm.nif.gov/31526810/

37. Zychowski KE, Hoffmann AR, Ly HJ, et al. The effect of aflatoxin-B1 on red drum (Sciaenops ocellatus) and assessment of dietary supplementation of NovaSil for the prevention of aflatoxicosis. Toxins (Basel). [Internet] 2013; [Acceso 19 de julio de 2020] 5(9):1555-1573 Disponible en: fittps:/ pubmed.nc6inlm.nih.gov/24064717/

38. Velasco $M$, Zague V, Dario $M$, Nishikawa D, Pinto $C$, et al. Characterization and Short-Term clinical study of clay facial mask. Rev Ciênc Farm Básica Apl. [Internet] 2016; [Acceso 18 de julio de 2020] 37(1): Disponible en: https://www. researchgate.net/publication/318508286_Characterization_and Short-Term_clinical_study_of_clay_facial_mask

39. Epstein WL. Topical prevention of poison ivy/oak dermatitis. Arch Dermatol [Internet] 1989; [Acceso 18 de julio de 2020] 125(4):499-501; Disponible en: https://pubmed.nc6i. nlm.nif.gov/2522756/\#: : text=Bentonite\%2C\%20kaolin\%2C\%20 and $\% 20$ silicone $\% 20$ gave, and $\% 20$ deserves $\% 20$ further\%20clinical\%20 evaluation.

40. Mahmoudi $M$, Adib-Hajbaghery $M$, Mashaiekhi $M$. Comparing the effects of Bentonite \& Calendula on the improvement of infantile diaper dermatitis: $A$ randomized controlled trial. Indian J Med Res. [Internet] 2015; [Acceso 18 de julio de 2020] 142(6):742-746; Disponible en: fttps:// pubmed.nc6inlm.nif.gov/26831423/

41. Ducrotte P, Dapoigny M, Bonaz B, Siproudhis L. Symptomatic efficacy of beidellitic montmorillonite in irritable bowel syndrome: a randomized, controlled trial. Aliment. Pharmacol. Ther. [Internet] 2005; [Acceso 18 de julio de 2020] 21: 435-44 Disponible en: https://onlinelibrary. wiley.com/ doi/full/10.1111/j.1365-2036.2005.02330.x

42. Chang FY, Lu CL, Chen CY, Luo JC. Efficacy of dioctahedral smectite in treating patients of diarrhea-predominant irritable bowel syndrome. J Gastroenterol Hepatol. [Internet] 2007; [Acceso 18 de julio de 2020] 22(12): 2266-2272: Disponible en: https://pubmed.nc6inlm.nif.gov/17559369/ 